\title{
BRIEF COMMUNICATION OPEN Biological pathways modulated by antipsychotics in the blood plasma of schizophrenia patients and their association to a clinical response
}

\author{
Daniel Martins-de-Souza ${ }^{1,2}$, Fiorella A Solari ${ }^{3}$, Paul C Guest ${ }^{1}$, René P Zahedi ${ }^{3}$ and Johann Steiner ${ }^{4,5}$
}

Proteomics is a valuable tool to unravel molecular mechanisms involved in human disorders. Considering the mediocre effectiveness of antipsychotics, which are the main class of drug used to treat schizophrenia, we analyzed a cohort of 58 schizophrenia patients who had blood collected before and after 6 weeks of antipsychotic treatment using a shotgun mass spectrometry proteomic profiling approach. Our aim was to unravel molecular pathways involved with an effective drug response. The results showed that all patients had essentially the same biochemical pathways triggered Independent of the antipsychotic response outcome. However, we observed that these pathways were regulated in different directions in blood samples from those who responded well to antipsychotics, compared with those who had a poorer outcome. These data are novel, timely and may help to guide new research efforts in the design of new treatments or medications for schizophrenia based on biologically relevant pathways.

npj Schizophrenia (2015) 1, Article number: 15050; doi:10.1038/npjschz.2015.50; published online 9 December 2015

Schizophrenia is the result of a combined dysfunction of genetic, biochemical and neurodevelopmental components that may be triggered by environmental factors. This cascade of events will most likely lead to disruption of cellular and tissue homeostasis, impairing the molecular pathways, which govern the cellular machinery throughout the body. Given the widespread nature of these effects, multiplex molecular approaches such as proteomics are required to provide new insights, as such methods can target hundreds of disease-relevant molecules, including those involved in the pathogenesis. In addition, by identifying specific proteins that are present at different steady-state levels in the disease state, proteomics can aid the discovery of molecular biomarker candidates. $^{1}$

Although our understanding of the molecular basis of schizophrenia has evolved recently, there are still many factors that do not connect or are still unknown. Thus, identification of these factors and increasing our understanding of their impact on the disease would be an important step forward. In addition, no factors emerging from molecular studies have been translated into clinical use for schizophrenia studies to date, despite the urgent need for both disease- and medication-related biomarker candidates. This is of particular importance owing to the fact that schizophrenia is an incurable disorder, which demands continuous healthcare, thus generating lifelong suffering for the patients and substantial expenses on the health-care systems. In the US, treatment and management of schizophrenia was estimated at a cost of 62 billion dollars per year, in the year $2000 .^{2}$

Although psychosocial interventions are available, disease management is mainly based on treatment with antipsychotic medications. However, $\sim 40 \%$ of schizophrenia patients do not respond properly to these medications and $>60 \%$ end up abandoning treatment due to undesirable side effects. ${ }^{3}$ Consequently, the intellectual and cognitive capacities of the patients may worsen, making them incapable of functioning adequately in society, thereby producing a further socioeconomic burden.

Consistent with our lack of understanding of disease pathophysiology, knowledge on the effects of medication on metabolism and other molecular pathways in the patients is scarce. With this in mind, we have employed a proteomic approach in an attempt to increase the understanding of the molecular pathways affected in response to currently used atypical antipsychotic medications. It was of particular interest to identify biomarker candidates associated with a positive response. The availability of such early-response biomarkers could eventually be used to help diminish the duration of poor response periods, thereby diminishing disease severity and improving the outcomes for the patients.

The cohort studied in this investigation consisted of 58 acutely ill patients who received the atypical antipsychotic drugs olanzapine $(n=18)$, quetiapine $(n=14)$ or risperidone $(n=26)$ (Table 1). Blood samples were collected by intravenous puncture as previously described in the psychiatric Clinic of the University of Magdeburg (Germany). ${ }^{4}$ Citrate plasma samples were collected at baseline (T0), when patients were acutely ill and either antipsychotic-naive $(n=23)$ or antipsychotic-free for at least 6 weeks $(n=35)$, and after 6 weeks when all the subjects had been treated as inpatients (T6). Patients with other medical conditions such as type 2 diabetes mellitus, hypertension, cardiovascular or autoimmune diseases were excluded. In line with previous studies, ${ }^{5}$ the patients were separated into responders $(n=36)$ and

\footnotetext{
'Laboratory of Neuroproteomics, Department of Biochemistry and Tissue Biology, Institute of Biology, University of Campinas (UNICAMP), Campinas, Brazil; ${ }^{2}$ UNICAMP's Neurobiology Center, Campinas, Brazil; ${ }^{3}$ Leibniz Institut für Analytische Wissenschaften (ISAS), Dortmund, Germany; ${ }^{4}$ Department of Psychiatry and Psychotherapy, University of Magdeburg, Magdeburg, Germany and ${ }^{5}$ The Center for Behavioral Brain Sciences, Magdeburg, Germany.

Correspondence: D Martins-de-Souza (dmsouza@unicamp.br)
}

Received 8 July 2015; revised 22 October 2015; accepted 28 October 2015 


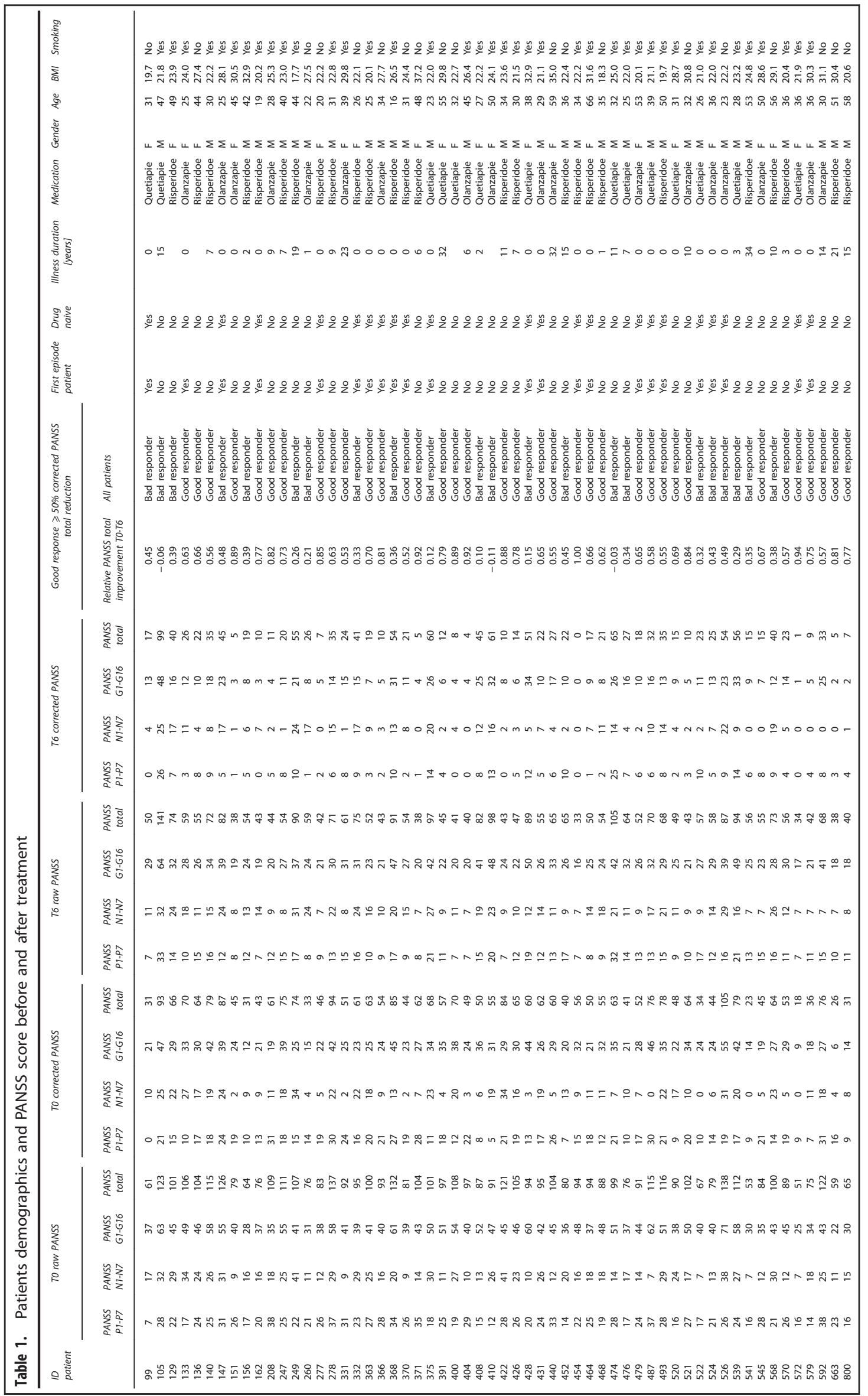


a

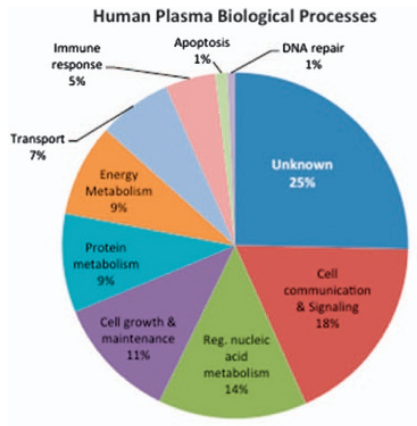

b

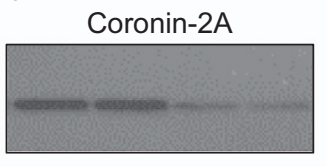

T6-R T6-R T6-NR T6-NR

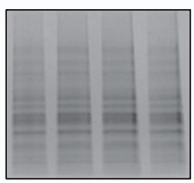

Loading control

C

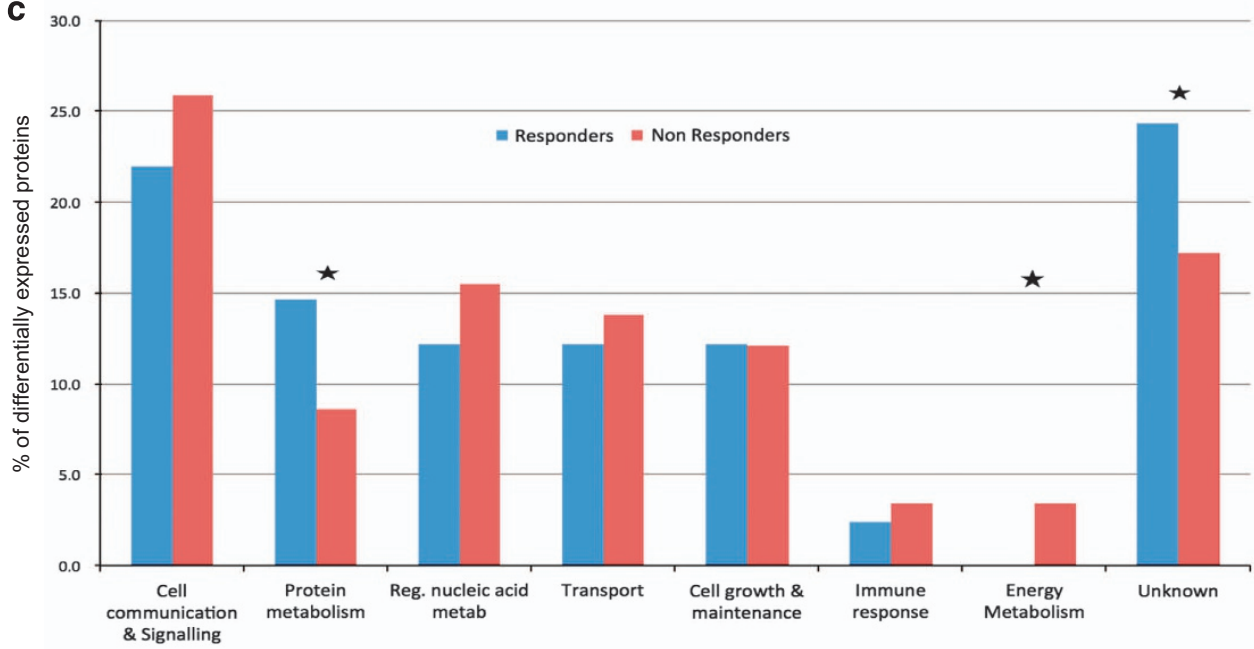

d

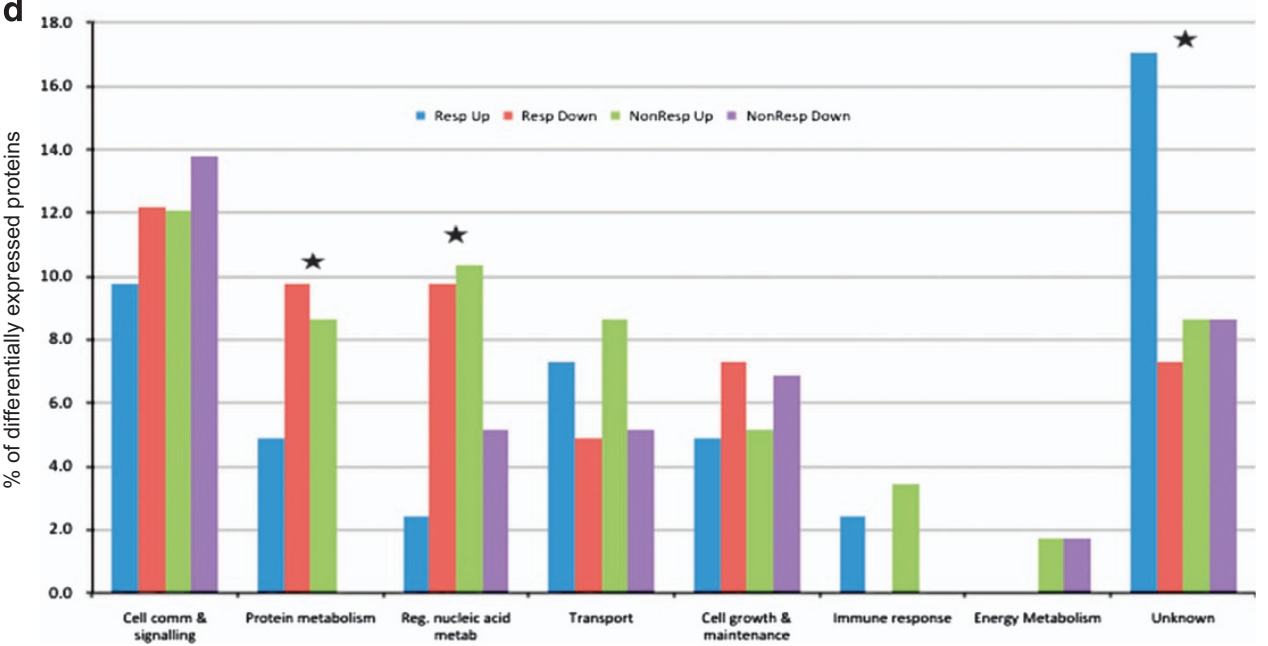

Figure 1. (a) Biological processes represented by all 985 plasma proteins identified through the shotgun mass spectrometry profiling approach; (b) western blot validation of the mass spectrometry findings for coronin-2A, which showed increased levels in responders and decreased levels in non-responders; (c) Biological pathways affected by antipsychotics in responders (blue bars) and non-responders (red bars); (d) Percentage of proteins increased in responders (blue), decreased in responders (red), increased in non-responders (green) and decreased in non-responders (purple) in the same biological pathways shown in part (c). For (c and d) the $y$ axis represents the percentage of differentially expressed proteins associated to those processes and a star indicates significant differences of the protein constituents (c) or directional changes (d) within a pathway.

non-responders $(n=22)$, with response defined as a $50 \%$ reduction of the corrected (subtraction of minimum scores representing 'no symptoms') total Positive and Negative Syndrome Scale (PANSS) scores. The percentage of responders was similar in the antipsychotic-naive (61\%; 14/23) and antipsychotic-free $(63 \% ; 22 / 35)$ subgroups (Table 1$)$.
Blood samples were immediately centrifuged for $10 \mathrm{~min}$ at $2,000 \times g$ and resulting supernatants divided in $250-\mu \mathrm{l}$ aliquots, which were immediately frozen at $-80^{\circ} \mathrm{C}$. Protein concentrations were determined by Bradford assay. Next, the plasma samples were depleted of 14 high-abundant proteins using the MARS-14 immunodepletion system (Agilent; Wokingham, UK) ${ }^{6}$ as these 


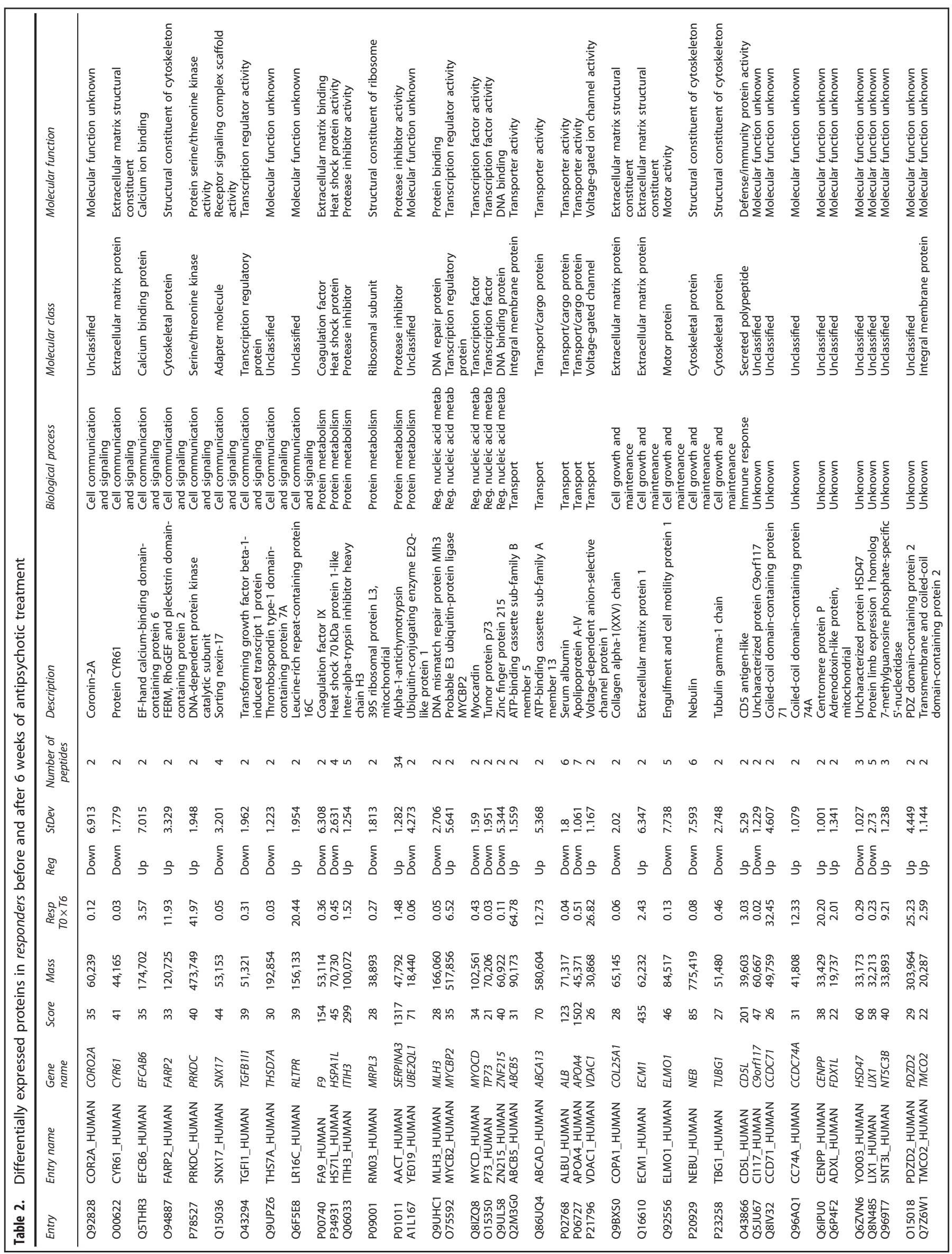




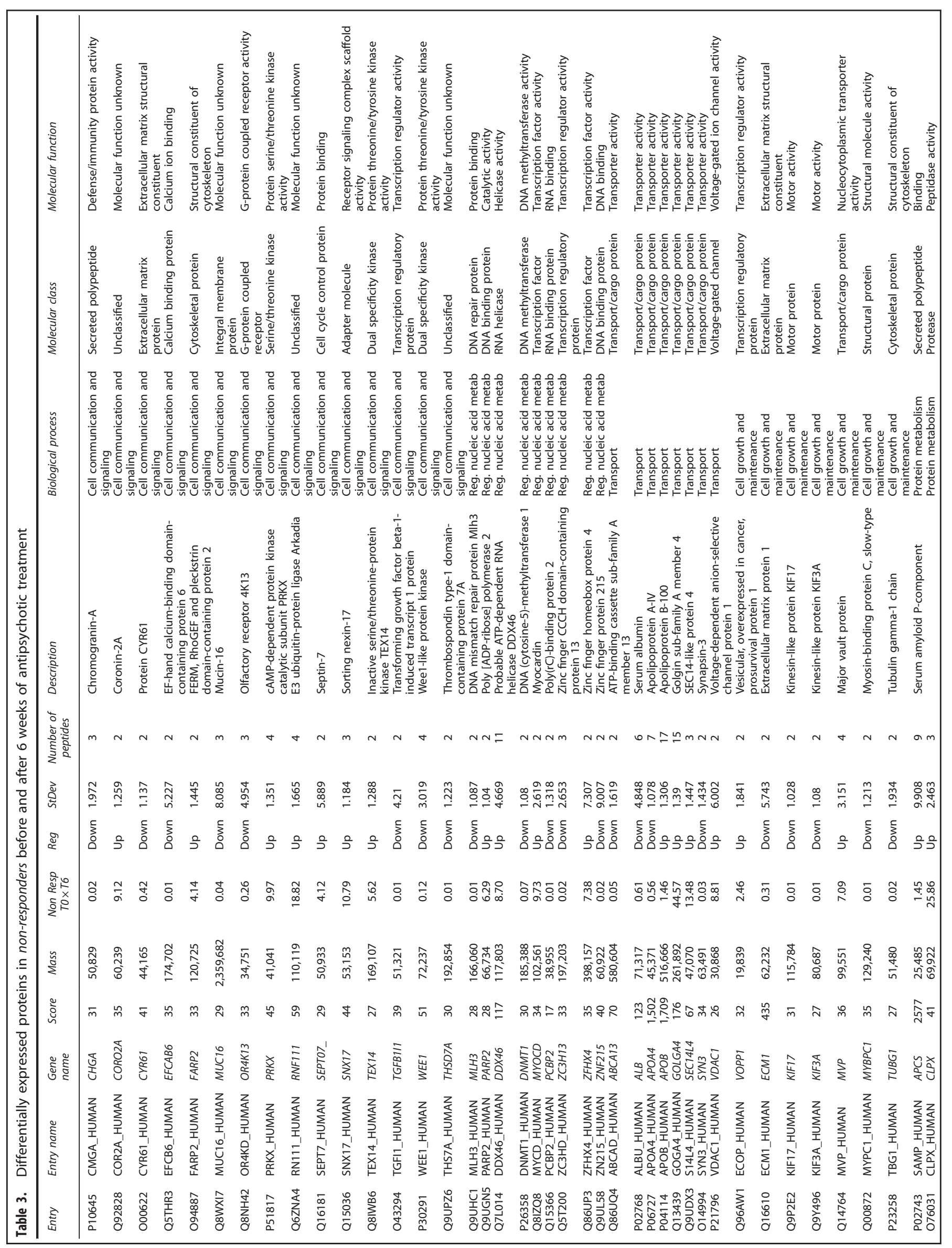


tend to obscure lower abundance proteins in proteomic studies. Flow-through fractions containing mostly low abundant proteins were treated successively with $5 \mathrm{mM}$ dithiothreitol $(30 \mathrm{~min}$, room temperature) and $10 \mathrm{mM}$ iodoacetamide $\left(30 \mathrm{~min}, 60^{\circ} \mathrm{C}\right.$ in the dark) to block reactive sulfhydryl groups on the proteins. The proteins in the samples were then enzymatically digested using trypsin (Promega, Heidelberg) at a trypsin:protein ratio of 1:50 for $16 \mathrm{~h}$ at $37^{\circ} \mathrm{C}$.

The peptides resulting from the trypsin digestion were analyzed using an Ultimate 3000 Rapid Separation Liquid Chromatography system (Dionex, Amsterdam, The Netherlands) coupled to an Orbitrap Elite mass spectrometer (Thermo Scientific, Bremen, Germany). For the chromatography stage, $1 \mu \mathrm{g}$ of peptide mixture from each sample was preconcentrated on a 100- $\mu \mathrm{m}$ trapping column (Acclaim C18 PepMap100, $100 \mu \mathrm{m} \times 2 \mathrm{~cm}$, Thermo Scientific) at a flow rate of $20 \mu \mathrm{l} / \mathrm{min}$ in $0.1 \%$ trifluoroacetic acid. This was followed by separation on a $75-\mu m$ column (Acclaim C18 PepMap100, $75 \mu \mathrm{m} \times 50 \mathrm{~cm}$, Thermo Scientific) using a binary gradient of solvents A $(0.1 \%$ formic acid) and B $(0.1 \%$ formic acid in $84 \%$ acetonitrile) ranging from $3 / 97 \% A / B$ to $58 / 42 \% A / B$ over $160 \mathrm{~min}$ at a flow rate of $250 \mathrm{nl} / \mathrm{min}$. In order to minimize carry-over effects from previous sample runs, a wash program with high organic content was used prior to each sample injection.?

For the mass spectrometry stage, samples were measured in data dependent acquisition mode, survey scans were acquired in the Orbitrap at resolution 60,000 and the 10 most intense signals were fragmented in the ion trap using collision-induced dissociation (CID). The respective target values and maximum injection times were $10^{6}$ ions and $100 \mathrm{~ms}$ for the MS stage, and $10^{4}$ ions and $50 \mathrm{~ms}$ for the MS/MS stage. Precursor ions with charge states between +2 and +5 were selected for fragmentation, using a dynamic exclusion of $15 \mathrm{~s}$.

The resulting raw data were processed using an in-house version of the MASCOT search engine for protein identification and MASCOT Distiller for label-free spectral counting quantification. The cut-off criteria were set at a minimum of 2 peptides for identification and at least $5 \mathrm{MS} / \mathrm{MS}$ spectra for quantification. Differences in protein expression between T6 and T0 samples for each patient were determined using Student's $t$-test $(P<0.05)$ with a false discovery rate set at 0.1. Differentially expressed proteins were classified according to their biological and molecular processes using the Human Protein Reference Database (http://www.hprd.org). For interpretation of the biological and biochemical significance of differentially expressed proteins, the UniProt identification codes of proteins with a T6/T0 ratio $>2: 1$ $(P>0.05)$ were uploaded into the Ingenuity Pathways Knowledgebase (IPKB). This software determines over-represented pathways by overlaying experimental protein data onto pre-existing biological pathway maps. The shotgun mass spectrometry profiling analysis resulted in identification of 18,227 peptides, corresponding to 985 proteins associated with 10 different biological processes (Figure 1a). Intriguingly, $25 \%$ of the proteins are of unknown function and are under investigation by our group.

Samples results were divided in four groups. These were T0 responders (T0-R), T6 responders (T6- $\mathrm{R})$, T0 non-responders (T0-NR) and T6 non-responders (T6-NR), independent of the antipsychotic employed. The comparison T6-R/TO-R resulted in identification of 41 differentially expressed proteins, which ranged across seven biological processes (Table 2). The comparison T6-NR/T0-NR identified 58 differentially expressed proteins, which covered eight biological processes (Table 3 ). One of the proteins (coronin-2A) was increased in responders and decreased in nonresponders. We evaluated the differential expression of coronin$2 \mathrm{~A}$ by western blot as described previously ${ }^{8}$ in two pools of the same samples analyzed by mass spectrometry to confirm this was not technically biased. The mass spectrometry finding was validated using a primary antibody against coronin-2A (Pierce, 
PA5-30206) (Figure 1b). The blotting membrane was stained with Ponceau Red and used as loading control.

In general, responders and non-responders showed similar effects on the same biochemical pathways after 6 weeks of antipsychotic treatment (Figure 1c). However, there were some differences as responders showed changes in almost twice the number of proteins involved in protein metabolism pathways, non-responders showed energy metabolism differences, which were not observed in the responders.

Potentially the most important finding of this study was that antipsychotic treatment led to opposite directional changes in some of the component proteins in responders and non-responders, as described for coronin-2A above. For example, the responders showed a 4.1 to 1 ratio of decreased to increased proteins in the pathway 'regulation of nucleic acids metabolism', and this ratio was 2.0 to 1 in the non-responders (Figure 1c and d). A similar scenario was observed for altered proteins associated with 'protein metabolism'. Although most of the protein components of this pathway showed a decrease in responders, all of the proteins of this same pathway were increased in non-responders.

Proteins regulating nucleic acids metabolism modulated by antipsychotics may be pivotal to processes such as DNA methylation and demethylation as well as chromatin remodeling, which may balance gene expression in response to antipsychotics. ${ }^{9}$ The differences we observed here reinforce the notion that the success of antipsychotic medication may be dependent on individual genetic backgrounds. In this scenario, the absorption, transport, receptor binding, metabolism and excretion of antipsychotics will be distinct for each patient. This supports the concept of personalized medicine strategies using pharmacogenomic approaches as a means of maximizing the potential effects of medications. ${ }^{10}$ In this study, antipsychotic treatment also modulated proteins associated with the 'metabolism of proteins' pathway oppositely in responders and non-responders. This is most likely related to protein turnover effects and may explain some of the above differences in protein expression levels in the two groups, as described in a previous study of antipsychotic treatment response (Lester et al. 2012).

The present results indicate that classifying responders and non-responders on the level of biochemical pathways rather than individual proteins may lead to a better understanding of the diverse effects of antipsychotics in schizophrenia patients. Investigation of individual proteins may not be as useful considering the diverse nature of human samples and the existence of redundant systems for the regulation of biochemical pathways in most biological systems. Nevertheless, several of these proteins such as coronin-2A are currently undergoing further analysis in our laboratory as potential antipsychotic treatment response biomarkers. Besides the added value to strategies of translational and personalized medicine, our results suggest new directions to be taken in the search for more effective treatments and the development of new medications for individuals suffering from schizophrenia.

\section{ACKNOWLEDGMENTS}

We dedicate our efforts to psychiatric patients and their families and thank them for understanding the importance of science and for having made our research possible. DMS is funded by FAPESP (São Paulo Research Foundation, grants 2013/08711-3, 2013/25702-8 and 2014/10068-4).

\section{CONTRIBUTIONS}

DMS and JS conceived the study. JS collected all samples from patients and clinical data. FAS and RPZ performed the mass spectrometry experiments. DMS obtained and analyzed the data and wrote the first version of the manuscript. FAS, RPZ and JS reviewed the data analysis and contributed significantly to manuscript writing. PCG edited the final version of the manuscript and contributed to data interpretation. All authors approved this version for publication.

\section{COMPETING INTERESTS}

The authors declare no conflict of interest.

\section{REFERENCES}

1. Martins de Souza D. Proteomics tackling schizophrenia as a pathway disorder. Schizophr. Bull. 38, 1107-1108 (2012).

2. Jablensky A. Epidemiology of schizophrenia: the global burden of disease and disability. Eur. Arch. Psychiatry Clin. Neurosci. 250, 274-285 (2000)

3. Tandon R., Nasrallah H. A., Keshavan M. S. Schizophrenia, 'Just the Facts' 5 treatment and prevention Past, present, and future. Schizophr. Res. 122, $1-23(2010)$

4. Steiner, J. et al. Increased prevalence of diverse N-methyl-D-aspartate glutamate receptor antibodies in patients with an initial diagnosis of schizophrenia: specific relevance of IgG NR1a antibodies for distinction from N-methyl-D-aspartate glutamate receptor encephalitis. JAMA Psychiatry 70, 271-278 (2013).

5. Leucht, S., Davis, J. M., Engel, R. R., Kissling, W. \& Kane, J. M. Definitions of response and remission in schizophrenia: recommendations for their use and their presentation. Acta Psychiatr Scand Suppl 119, 7-14 (2009).

6. Jaros, J. A., Guest, P. C., Bahn, S. \& Martins de Souza, D. Affinity depletion of plasma and serum for mass spectrometry-based proteome analysis. Methods Mol. Biol. 1002, 1-11 (2013).

7. Burkhart, J. M., Schumbrutzki, C., Wortelkamp, S., Sickmann, A. \& Zahedi, R. P. Systematic and quantitative comparison of digest efficiency and specificity reveals the impact of trypsin quality on MS-based proteomics. J. Proteomics 75, 1454-1462 (2012).

8. Guest, P. C. et al. MK-801 treatment affects glycolysis in oligodendrocytes more than in astrocytes and neuronal cells: insights for schizophrenia. Front. Cell Neurosci. 9, 180 (2015).

9. Guidotti, A. \& Grayson, D. R. DNA methylation and demethylation as targets for antipsychotic therapy. Dialogues Clin Neurosci 16, 419-429 (2014).

10. Xu, Q. et al. Pharmacogenomics can improve antipsychotic treatment in schizophrenia. Front. Med. 7, 180-190 (2013).

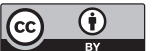

This work is licensed under a Creative Commons Attribution 4.0 International License. The images or other third party material in this article are included in the article's Creative Commons license, unless indicated otherwise in the credit line; if the material is not included under the Creative Commons license, users will need to obtain permission from the license holder to reproduce the material. To view a copy of this license, visit http://creativecommons.org/licenses/ by/4.0/ 